\title{
Determination of relationship between breastfeeding self- efficacy of mothers and nipple pain/trauma
}

\author{
Serpil Özkul and Esin Çeber Turfan* \\ Izmir Atatürk School of Health, Ege University, Izmir, Turkey
}

\begin{abstract}
Aim: In the first months of lactation, nipple pain and chaps emerge among most of the mothers due to inadequate breastfeeding, and it gets difficult for the baby to feed on breast milk. Ineffective breastfeeding and baby feeding in wrong position cause damage, chap of nipple and pain while breastfeeding and mothers may give up breastfeeding for this reason. Therefore, using scales which measure efficacy of breastfeeding is important in order to predetermine nipple pain and trauma risk and prevent formation of nipple chaps. When the risk is determined, there would be a chance of removing the problem before it occurs through suitable midwifery care and breastfeeding.

In this study, it was aimed to determine the relation between self-efficacy of breastfeeding of mother and nipple pain/trauma.

Methods: In data collection of this study which is a sectional and analytical research, a question form was used about descriptive characteristics of puerperant, 'LATCH Breastfeeding Charting Scale' and 'Breastfeeding Self-Efficacy Scale' were used in order to evaluate breastfeeding efficacy; 'Nipple Pain and Trauma' was used in order to determine nipple pain and trauma of puerperant mothers.

Setting and study participants: The study was carried out at Gerbay Family Health Center in İzmir-Bayrakl1. Puerperants $(74)$ within $8^{\text {th }}$ and $40^{\text {th }}$ days of puerperium period were included in the study since nipple pain and chap are observed in the first weeks of postnatal period.

Findings: According to Breastfeeding Self-efficacy Scale, although all of the puerperants regard themselves as 'efficacious' abut breastfeeding; when their breastfeeding efficacy are evaluated with LATCH scale it was determined that $83.8 \%$ of them are 'inefficacious' about breastfeeding and do not breastfeed according to technique. Moreover, it was determined that $77 \%$ of puerperants who do not use suitable breastfeeding technique have nipple chap.

Results and Suggestions: Study results are important since they show that although mothers regard themselves efficacious about breastfeeding they are in fact inefficacious when evaluated with an objective scale and in this sense they have nipple chap. Therefore, in puerperium period especially midwives and nurses should observe and examine breastfeeding techniques and positions; if there is a problem on nipple it should be determined and solved at an early period
\end{abstract}

\section{Introduction}

Breastfeeding is the most suitable and unique feeding method for health growth and development of babies. It is also a communication tool which has biological and emotional effect on the health of mother and baby [1]. However, in the first months of lactation, nipple pain and chaps emerge among most of the mothers due to inadequate breastfeeding, and it gets difficult for the baby to feed on breast milk. Nipple pain and chaps sometimes reach an extent that would prevent breastfeeding of mothers and baby to make use of breast milk. In a study, it was determine that $80-90 \%$ of breastfeeding mothers experience nipple pain and $26 \%$ of these problems progress and turn into nipple chaps and quite serious nipple pain [2]. Moreover, nipple chaps increase in the following days among women who have nipple pain and rash in postnatal period [3]. Nipple pain and chaps result from skin types of mother, nipple type, lack of nutrition; as well as breastfeeding techniques and problems such as wrong breastfeeding position, milk accumulating in the breast, frequency and duration of breastfeeding [4]. Protective measures can be taken together with supporting breastfeeding, since it would pose risk for nipple pain and trauma due to low breastfeeding efficacy. After nipple chaps occur, various pomades or creams are suggested for its treatment. However, it is stated that treatments are not so effective once the chaps occur
[5]. Therefore, before any nipple pain or chaps occur, determination of relation between breastfeeding status and efficacy of mother and nipple pain and chap is a valuable data for showing the need for breastfeeding training to be provided for mothers. In post natal period, breastfeeding efficacy of mother should be evaluated with objective scales and risky conditions in the sense of nipple pain and chap should be determined. Although various tools are used in our country in order to measure sufficiency and efficacy of breastfeeding, there is no study about effectiveness of this scale in determining breastfeeding and risk of nipple pain and trauma. In this study; breastfeeding efficacy of puerperants were defined by using Breastfeeding Charting Scale (LATCH) and Breastfeeding Self-efficacy Scale. The relationship between nipple pain and trauma was analyzed with Breastfeeding efficacy and breastfeeding self-efficacy scales, and it was suggested that breastfeeding training should be developed accordingly.

${ }^{\star}$ Correspondence to: Esin Ceber, Associate Professor, Izmir Atatürk School of Health, Ege University, Izmir, Turkey, Tel: +902323882851; E-mail: esin.ceber@ ege.edu.tr

Key words: breastfeeding, efficacy, nipple pain, nipple trauma

Received: June 02, 2018; Accepted: June 26, 2018; Published: June 30, 2018 


\section{Material and Method}

This study which was carried in order to determine the relationship between breastfeeding efficacy status of mothers and nipple pain/ trauma is sectional, analytical field study.

The study was carried out in Gerbay Family Health Center no.14 in Bayrakl, İzmir. Instead of all the mothers living and breastfeeding in the region, only those who are within $8^{\text {th }}$ and $40^{\text {th }}$ days of puerperium were included in the study since nipple pain and chaps are observed in the first weeks in postnatal period. It was aimed that the universe of study was composed of all puerperants (N: 110) who are registered at Bayraklı Gerbay Family Health Center no.14 which serves for a vast population. Puerperants who have given birth between 15 November 2009 and 15 March 2010, who have the criteria for participating in and who accepts being participated after being informed about study were included in the study. As a result of power analysis, the number puerperants who are to be included in the sampling was determined as 71 by using $0.95, \beta: 0.04$ and $\alpha: 0.05 .74$ puerperants were accessed in this study. Data of the study were collected with home visit and face to face interview with puerperants who are registered at Gerbay FHC no14 in Bayrakl1, İzmir between 15.11.2009 - 15.03.2010.

Within the process of study, four different data collection forms were used; a question form about descriptive properties of puerperants, two scales to evaluate breastfeeding efficacy and an algorithm to determine nipple pain and trauma. Descriptive characteristics question form is composed of 46 items in order to determine descriptive characteristics of mothers. LATCH Breastfeeding Charting Scale was used in order to determine breastfeeding efficacy of puerperant mothers objectively. 0 , 1,2 scores are given for each criteria in LATCH Breastfeeding Charting Scale. Breastfeeding is evaluated after all the scores are summed up. If the total score counts 10 it means breastfeeding is 'efficacious', if it is below 10 then it is 'inefficacious' (which means mother needs help) [6,7]. In the study Breastfeeding Self-efficacy Scale which was developed by Cindy-Lee Dennis in order to measure breastfeeding efficacy, adapted into Turkish by Ekşioğlu and Çeber and composed of 33 items was used in order to determine how mothers evaluate their own breastfeeding efficacy [8].

In the fourth phase, Nipple and Trauma Algorithm was used in home visits between $8^{\text {th }}$ and $40^{\text {th }}$ days of puerperium period. This algorithm was translated from English and Turkish by five experts. Language validity of the algorithm was determined by consulting to the view of five experts, (Kendall Coefficient of Concordance) (W) correlation test was done in order to evaluate questions in the algorithm are compatible with the aim of measurement for content validity, W: $\mathbf{0 . 2 8 7}$ obtained in Kendall Coefficient of Concordance, it was determined that there is statistical compatibility between expert views ( $\mathrm{p}>0.05$ ). 'Nipple pain and trauma algorithm' classifies nipple chap stages in 5 different ways. However, in the study, while nipple pain and trauma are evaluated, classification was reduced to 3. In classification of nipple pain and trauma, 1. degree was classified as 'no nipple chap'; 2. degree was classified as 'surface chap' 3. degree was classified as 'deep chap'. While the relationship between Breastfeeding Efficacy Scale Score and Nipple Pain and Trauma is analyzed, this grading was reduced to two in statistical analyses; 'no nipple chap' (those who have nipple chap and surface damage), 'nipple chap' (those who have surface chap+deep chap on the nipple) and chi-square analysis was done.

In the analysis of data, percentage distribution, average, standard deviation, Chi-square and correlation analyses were done.
Women who were included in the study sample were informed about the aim of study and participation to the study and their consent were taken.

\section{Findings}

$50 \%$ of the puerperants who are included in the study are in 25-29 age group, their age average is $27.4 \pm 0.93(\min =18.0, \max =41.0)$. it was determined that $51,4 \%$ of the puerperants are elementary school graduates, $83,8 \%$ do not work and are housewives, 53,5\% are multipara mothers. It was stated that $75.7 \%$ of the pregnancies are planned. According to the type of birth giving, $62,2 \%$ stated that they have given caesarean birth.

When the first time of breastfeeding following birth was asked to puerperants, it was determined that $64,9 \%$ breastfed their baby within the first 30-60 minutes following birth. When breastfeeding experience of puerperants were analyzed, $54,1 \%$ stated they have never breastfed any baby and this is their first experience, $70,3 \%$ stated they have never received any breastfeeding training.

When breastfeeding efficacy of puerperants were evaluated according to LATCH Breastfeeding Charting Scale, it was determined that $83,8 \%$ are 'inefficacious' in breastfeeding and do not carry out the act according to suitable technique (Table 1).

In the sense of breastfeeding self-efficacy scale and personal attitude and perception, it was determined that puerperants regard themselves as efficacious (100\%) during breastfeeding, this case increased breastfeeding self-efficacy.

It was determined that there is statistically significant relation between LATCH Breastfeeding Efficacy Scale score which evaluate breastfeeding objectively and Breastfeeding Self-efficacy in which puerperants evaluate their own breastfeeding efficacy ( $\mathrm{r}: 0.557, \mathrm{p}<$ 0.05 ) and while LATCH score increases breastfeeding self-efficacy score increases as well (Table 2, Figure 1).

According to nipple pain and trauma, those who have surface chap and deep chap on the nipple $(70.3 \%)$ was evaluated as 'nipple chap exists'; those who have no surface damage on nipple (23\%) was evaluated as 'no nipple chap' (Table 3).

According to LATCH breastfeeding efficacy scale, it was determined that $91,7 \%$ of puerperants who are regarded as efficacious do not have nipple chap $\left(X_{F}^{2}: 38.19, \mathrm{p}<0.01\right)$. It was determined that nipple chap is more common among puerperants whose breastfeeding self-efficacy scores are insufficient (Table 4).

When the difference between nipple pain and trauma are analyzed according to descriptive characteristics of puerperants, it was determined that nipple pain and trauma are more common among puerperants in 20-29 age group. It was determined that there

Table 1. LATCH Breastfeeding Charting Scale Scores of Puerperants and Distribution according to Efficacy

\begin{tabular}{|c|c|c|}
\hline LATCH Scale Score & Number & \% \\
\hline $0-9$ (inefficacious) & 62 & 83.8 \\
\hline 10 (efficacious) & 12 & 16.2 \\
\hline Total & 74 & 100.0 \\
\hline
\end{tabular}

Table 2. LATCH score increases breastfeeding self-efficacy score increases

\begin{tabular}{|c|c|}
\hline & Breastfeeding Self-efficacy \\
\hline LATCH & $\mathrm{P}=0.000$ \\
$\mathrm{r}=0.557(* *)$
\end{tabular}




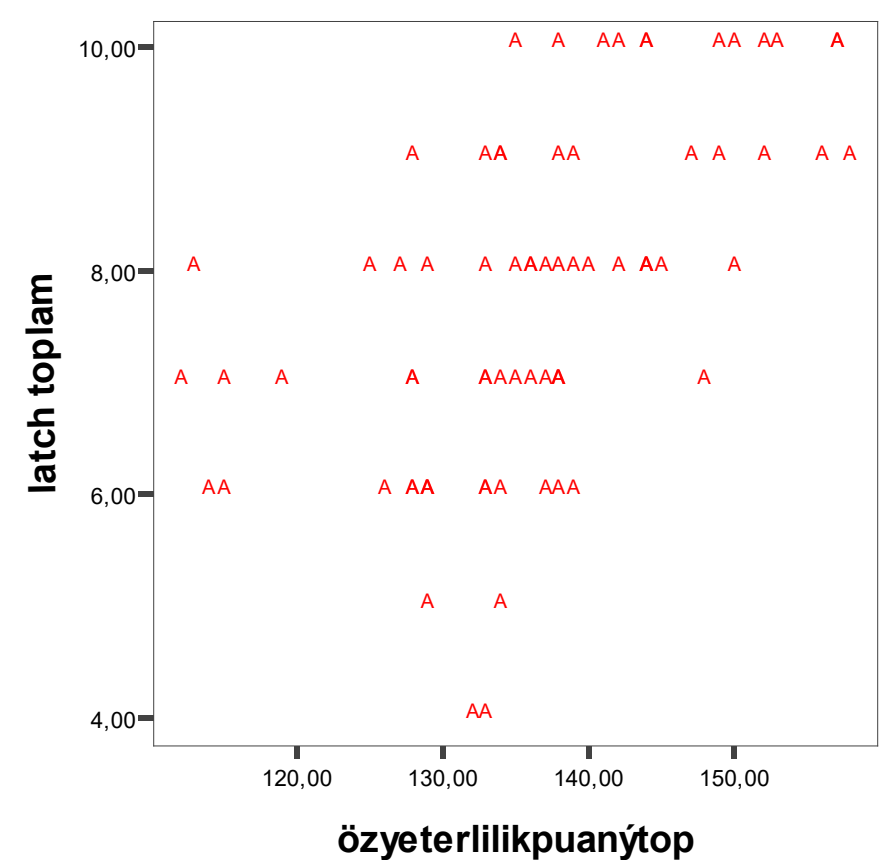

Figure 1. The Relationship between LATCH Breastfeeding Status of Puerperants and Breastfeeding Self-Efficacy

Table 3. Distribution of Puerperants according to 'Nipple Pain and Trauma Classification'

\begin{tabular}{|l|l|l|}
\hline $\begin{array}{l}\text { Nipple Pain and Trauma (Algorithm } \\
\text { Classification) }\end{array}$ & Number & $\mathbf{\%}$ \\
\hline No nipple chap & 17 & 23.0 \\
\hline Surface chap & 35 & 47.3 \\
\hline Deep chap & 22 & 29.7 \\
\hline Total & 74 & 100.0 \\
\hline
\end{tabular}

Table 4. Nipple Pain and Chap Status of Puerperants according to Breastfeeding Efficacy (LATCH)

\begin{tabular}{|c|c|c|c|c|c|c|c|c|}
\hline & \multicolumn{4}{|c|}{ Nipple chap } & & & \multirow{2}{*}{$\mathrm{X}_{\mathrm{F}}^{2}$} & \multirow[b]{3}{*}{0.000} \\
\hline & \multicolumn{2}{|c|}{ Yes } & \multicolumn{2}{|c|}{ No } & & & & \\
\hline & Numbe & $\%$ & Number & $\%$ & Number & $\%$ & $38.19 *$ & \\
\hline Efficacious & 1 & 8.3 & 11 & 91.7 & 22 & 100.0 & & \\
\hline Inefficacious & 56 & 90.3 & 6 & 9.7 & 62 & 100.0 & & \\
\hline Total & 57 & 77.0 & 17 & 23.0 & 74 & 100.0 & & \\
\hline
\end{tabular}

is statistically significant difference between nipple pain and trauma according to age groups $\left(\mathrm{X}^{2}: 4.30, \mathrm{p}<0,05\right)$. When nipple pain and chap frequency among primiparous and multipara mothers is analyzed, it was determined that there is no nipple chap among $14,8 \%$ of primiparous mother and $27,7 \%$ of multipara mothers.

\section{Discussion}

In the study, according to Breastfeeding Self-efficacy Scale, although all of the puerperants regard themselves as 'efficacious' abut breastfeeding; when their breastfeeding efficacy are evaluated with LATCH scale it was determined that $83.8 \%$ of them are 'inefficacious' about breastfeeding and do not breastfeed according to technique. It was determined that as breastfeeding Self-efficacy score increases, so does Breastfeeding Efficacy (LATCH) score. It was determined that nearly $1 / 3$ of the puerperants have nipple chap; nipple chap frequency decreases among those who are efficacious in breastfeeding.

In the study it was determined that $77 \%$ of puerperants have nipple chap. In previous studies which was carried out with breastfeeding mothers, it was determined that nipple chap frequency varies between $11 \%$ and $96 \%[9,10]$. Similar to this study, it was determined in another study of Ziemer, et al. [11] that $80-95 \%$ of mothers have nipple pain, $26 \%$ of them have serious pain. In another study, similarly there is $34 \%$ nipple pain, $16 \%$ nipple chap, nipple pain and chap in total is 50\% [3]. That nipple pain problems are at high rates points out to the fact that nipple chap is an important issue in breastfeeding training.

According to LATCH breastfeeding efficacy scale, it was determined that $83.8 \%$ of puerperants are 'inefficacious' in breastfeeding and puerperants do not breastfeed according to technique. Similarly, in the study of Şahin, mothers were asked latching style of babies during breastfeeding, $40,8 \%$ of them answered that latching only nipple is enough which shows that they are inefficacious about breastfeeding technique. Nipple chap occurrence in the story of mothers who give the answer that baby should latch nipple is significantly higher compared to those who answered areola [12]. In the study of Ingram, et al. [13] breastfeeding problem of mother have decreased significantly in the second and sixth months once they have learned correct breastfeeding technique. In their study, Duffy, et al. [14] determined that when correct breastfeeding training is given to women in antenatal period, nipple pain, nipple trauma and breastfeeding rates decreased. This case is important since it points out that correct breastfeeding technique given one to one by midwives in antenatal and postpartum periods would be effective in reducing nipple pain and trauma. These findings show that use of correct technique in breastfeeding is important in preventing nipple chap.

Since Breastfeeding Self-efficacy Scale is a subjective scale which measures feelings and emotions of mother about breastfeeding, score of breastfeeding self-efficacy was found high among all the puerperants who participated in the study. In his study, Ekşioğlu determined as well that puerperants stated their breastfeeding self-efficacy status as highly efficacious [15]. It is clear that puerperants whose breastfeeding self-efficacy is high would be more sensible and willing about breastfeeding which would positively effect breastfeeding. In the study it was determined that there is statistically significant relation between LATCH Breastfeeding Efficacy Scale score and Breastfeeding Selfefficacy and while LATCH score increases breastfeeding self-efficacy score increases as well. This is a valuable finding since it points out that increasing self-confidence and self-efficacy in breastfeeding training would also increase their success in breastfeeding.

According to LATCH breastfeeding efficacy scale, it was determined that $91,7 \%$ of puerperants who are regarded as 'efficacious' do not have nipple chap. In this study according to LATCH breastfeeding efficacy scale, there is a difference in nipple pain and trauma status between puerperants who are efficacious in breastfeeding and who are not. In this study it was determined there is statistically negative relation between LATCH scale score and nipple and trauma. While breastfeeding LATCH score increase nipple pain and trauma decrease; LATCH score decreases while nipple pain and trauma increase. This finding is important since it shows that enabling efficacy to puerperants through breastfeeding training would prevent nipple chap.

It was determined that there is negative relation between breastfeeding self-efficacy scores of puerperants and nipple pain and trauma. It was determined that nipple pain and trauma decrease as breastfeeding self-efficacy score increases. In the study puerperants' having high self-perception of breastfeeding can be regarded as an important result since it would be effective in solving breast problems such as nipple pain and chap. When breastfeeding efficacy status $(\mathrm{LATCH})$ of primiparous and multiparous mothers are analyzed, it 
was determined that $23.4 \%$ of multiparous puerperants are 'efficacious', while $3.7 \%$ of primiparous puerperants are 'efficacious'. Similar to this study, in the study of Çakmak and Demirhan [16], it was determined that mothers who have second children are more successful in breastfeeding compared to mothers who have first children. In another study, nipple chap was observed nearly 3 times more commonly among primiparous mothers compared to multiparous mothers, and 11 times more commonly among mothers who breastfeed in wrong position.

When birth giving types of puerperants are analyzed, it was determined that there is no statistically significant difference between caesarean and normal birth in the sense of breastfeeding efficacy. In another study, similar to this one; it was stated that there is no statistically significant difference between averages of LATCH score of mother who have given normal spontaneous birth and who have given caesarean birth $[11,17]$. However; although there is no statistically significant difference between puerperants who have given normal and caesarean birth, it is an attractive result that breastfeeding efficacy among puerperant who have given normal birth doubles those who have given caesarean birth.

It was determined that there is statistically significant difference between breastfeeding experience and breastfeeding efficacy (LATCH) of puerperants. This case makes us think that having breastfeeding experience influence breastfeeding self-efficacy of them positively; enables them to feel efficacious about breastfeeding and decrease breastfeeding inefficacy since they are more conscious about correct breastfeeding (by using LATCH scale).

\section{Result}

As a result of study, it was determined that as breastfeeding efficacy (LATCH) of puerperants increase so does breastfeeding selfefficacy, there is a difference in nipple pain and trauma status between puerperants who are efficacious in breastfeeding and who are not according to LATCH breastfeeding scale, as breastfeeding self-efficacy of puerperants increase, their nipple pain and trauma decrease.

\section{Suggestions}

Midwives should examine breastfeeding technique of mothers, breastfeeding efficacy according to LATCH scale and breastfeeding self-efficacy status during visits in puerperium period, if there is any nipple problem it should be determined in the early stages, graded and treated. In this way, on one hand factors causing nipple chap would be determined and breastfeeding consultancy given for puerperant would be planned, on the other hand by preventing nipple pain and chap babies would receive breast milk more easily and longer.

\section{References}

1. Tezergil B (2007) Thoughts and practice of postnatal mothers about breastfeeding Post-graduate Thesis, Istanbul: Marmara University Maternity and Gynecological Diseases Nursing Department.

2. Melli MS, Rashidi MR, Nokhoodchi A, Tagavi S, Farzadi L, et al. (2007) A randomized trial of peppermint gel, lanolin ointment, and placebo gel to prevent nipple crack in primiparous breastfeeding women. Med Sci Monit 13: 9 .

3. GANS B (1958) Breast and nipple pain in early stages of lactation. Br Med J 2: 830832. [Crossref]

4. Walker M, Driscoll JW (1989) Sore nipples: the new mother's nemesis. MCN Am J Matern Child Nurs 14: 260-265. [Crossref]

5. Mohrbacher N, Stock J (1991) The Breastfeeding Answer Book, Illinois, La Lech League International, Innc.

6. Jensen D, Wallace S, Kelsay P (1994) LATCH: A breastfeeding charting system and documentation tool. J Obstet Gynecol Neonatal Nurs 23: 27-32.

7. Demirhan F (1997) Evaluation of Breastfeeding in Sakarya, Post-graduate Thesis. Istanbul: Marmara University SBE.

8. Dennis CL, Faux S (1999) Development and psychometric testing of the Breastfeeding Self-Efficacy Scale. Res Nurs Health 22: 399-409. [Crossref]

9. Centuori S, Burmaz T, Ronfani L, Fragiacomo M, Quintero S, et al. (1999) Nipple care, sore nipples, and breastfeeding: a randomized trial. J Hum Lact 15: 125-130. [Crossref]

10. Tait P (2000) Nipple pain in breastfeeding women: Causes, treatment, and prevention strategies. J Midwifery Womens Health 45: 212-215.

11. Ziemer MM, Pigeon JG (1993) Skin changes and pain in the nipple during the 1st week of lactation. J Obstet Gynecol Neonatal Nurs 22: 247-256. [Crossref]

12. Sahin G (2008) Evaluation of breastfeeding mothers' knowledge, practice and behavior about breastfeeding. Dissertation, Istanbul: Ministry of Health, Göztepe Training and Research Hospital, Family Medicine Coordinator.

13. Ingram J, Johnson D, Greenwood R (2002) Breastfeeding in Bristol: teaching good positioning, and support from fathers and families. Midwifery 18: 87-101. [Crossref]

14. Duffy EP, Percival P, Kershaw E (1997) Positive effects of an antenatal group teaching session on postnatal nipple pain, nipple trauma and breast feeding rates. Midwifery 13: 189-196.

15. Eksioglu AB, Ceber E (2011) Translation and validation of the Breast-feeding Selfefficacy Scale into Turkish. Midwifery 27: e246-253. [Crossref]

16. Çakmak H (2002) Evaluation and Comparison of Breastfeeding among Mothers Experienced Caesarean and Normal Spontaneous Birth. Post-graduate Thesis, Istanbul: Marmara University SBE.

17. Kumar SP, Mooney R, Wieser LJ, Havstad S (2006) The LATCH scoring system and prediction of breastfeeding duration. J Hum Lact 22: 391-397. [Crossref]

Copyright: (C2018 Özkul S. This is an open-access article distributed under the terms of the Creative Commons Attribution License, which permits unrestricted use, distribution, and reproduction in any medium, provided the original author and source are credited. 